\title{
Integrated simulation approach for laser-driven fast ignition
}

\author{
W.-M. Wang,, 1, , $*$ P. Gibbon, ${ }^{1}$ Z.-M. Sheng, ${ }^{3,4}$ and Y.-T. $\mathrm{Li}^{2}$ \\ ${ }^{1}$ Forschungzentrum Juelich GmbH, Institute for Advanced Simulation, \\ Juelich Supercomputing Centre, D-52425 Juelich, Germany \\ ${ }^{2}$ Beijing National Laboratory for Condensed Matter Physics, \\ Institute of Physics, CAS, Beijing 100190, China \\ ${ }^{3}$ Department of Physics, SUPA, Strathclyde University, \\ Rottenrow 10\%, G4 ONG Glasgow, United Kingdom \\ ${ }^{4}$ Key Laboratory for Laser Plasmas (MoE) and Department of Physics and Astronomy, \\ Shanghai Jiao Tong University, Shanghai 200240, China
}

(Dated: June 4, 2022)

\begin{abstract}
An integrated simulation approach fully based upon particle-in-cell (PIC) model is proposed, which involves both fast particle generation via laser solid-density plasma interaction and transport and energy deposition of the particles in extremely high density plasma. It is realized by introducing two independent systems in a simulation, where the fast particle generation is simulated by a full PIC system and the transport and energy deposition computed by a second PIC system with a reduced field solver. Data of the fast particles generated in the full PIC system are copied to the reduced PIC system in real time as the fast particle source. Unlike a two-region approach, which takes a single PIC system and two field solvers in two plasma density regions, respectively, the present one need not match the field-solvers since the reduced field solver and the full solver adopted respectively in the two systems are independent. A simulation case is presented, which demonstrates that this approach can be applied to integrated simulation of fast ignition with real target densities, e.g., 300 $\mathrm{g} / \mathrm{cm}^{3}$.
\end{abstract}

\section{INTRODUCTION}

As an alternative route to realize laser fusion energy, the fast ignition (FI) scheme has attracted significant attention since it was proposed by Tabak et al. two decades ago [1]. This scheme separates the compression and ignition processes and consequently relaxes the constraints of compression density and symmetry, which may offer the possibility of higher energy gain. A high coupling of energy has been demonstrated experimentally by Kodama et al. in 2001 [2]. In their experiment a metal cone is embed into the target to guide the propagation of fast electrons and the ignition laser as well as to reduce the distance of the electrons to the target core. Since then, many groups worldwide have performed studies on all aspects of FI physics including generation of fast electrons via laser plasma interaction, transport of the electrons in coronal plasma with steep density gradient, and heating of the target core [3-16]. Most studies have mainly focused upon one or two of these processes.

Integrated investigations of these processes are essential to fully assess the FI scheme. For this purpose, integrated experimental studies with large-scale FI targets are ideal and play a decisive role, however which need high energy laser beams and carefully designed targets, involving difficulties in both technology and physical understanding. Prior to such experiments, large-scale, integrated numerical simulations are a good choice. But it is hard to find a model to describe all the involved processes. Traditional particle-in-cell (PIC) models [17-19]

\footnotetext{
* Please also see this paper in Phys. Rev. E 91, 013101 (2015)
}

are suitable to simulate the generation of fast electrons from interaction of laser and plasma below and around $100 n_{c}$, where $n_{c}=1.1 \times 10^{21} \mathrm{~cm}^{-3}$ is the critical density corresponding to $1-\mu m$-wavelength lasers. It is almost impossible to apply this model to the problem of fast electron transport in FI plasma targets with the density growing from hundreds of $n_{c}$ to tens thousands of $n_{c}$ due to huge numerical noise appearing with unresolved plasma oscillation. A hybrid PIC model 20 23] has been employed to calculate the electron transport and energy deposition, in which the background plasma is considered as a fluid. In this case, the displacement current in Ampere's law is omitted and high frequency dynamics of the background plasma need not be resolved. Hence, this approach cannot describe laser plasma interaction.

To include all the three processes within an integrated model, Sentoku and Kemp proposed to artificially reduce the plasma density in collisional PIC simulation when it exceeds a upper-limit value, e.g., $500 n_{c}$ 24]. In this approach a macroparticle has two weights: one is its real weight which is used to calculate Coulomb collision and another is a reduced weight to calculate the current for the field solver. Hence, the electromagnetic (EM) fields are not consistent with the real plasma density, which can be applied when the resistive effect is much less than the collisions. To use this approach, one should make sure that the numerical noise at high density region is controlled to be quite low since the energy of a macroparticle with a reduced weight gaining from the noise is amplified by the ratio of the real weight to the reduced one. With this model Chrisman et al. have performed a group of integrated FI simulations with the core density as high as $20000 n_{c}$ or $100 \mathrm{~g} / \mathrm{cm}^{3}$ [25]. 
An improved model, named the two-region PIC, was proposed by Cohen et al. 26] in 2010, in which the high plasma density is not clamped artificially. In this model, the simulation box is separated into a low plasma density region and a high density region, where the density at the boundary between the two regions is taken as $\sim 100 n_{c}$. In the low density region a full PIC algorithm with collisions is taken and in the other region the Maxwell's equations are reduced by use of the Ohm's law to solve the electric fields while the Ampere's law is used to calculate currents of background electrons. This reduced field solver is similar to the one used in the hybrid PIC model [20 23], whereas the background plasma comprises macroparticles as in a traditional PIC model. In this case the EM fields are consistent with the plasma density in the whole simulation region. However, a potential challenge for this model arises in that the continuity of EM fields near the boundary of the two regions can be violated due to the noise of the full field solver. This noise is usually several orders of magnitude higher than the one of the reduced field solver, which may mask the real value given by the reduced field solver after a long period of simulation. Therefore, matching the EM fields around the boundary becomes challenging, although it may be partially solved by increasing the spatial resolution and using a large number of macroparticles per cell.

Here, we propose an approach to release the constraint of the EM field matching around the boundary of the two regions. Our approach involves two independent simulation systems: one using the full PIC model and another applying the reduced model as adopted before in the high density region of the two-region PIC approach [26]. Moreover both the full and reduced Maxwell's equations are solved in the whole simulation box, and the two solutions are independent in our approach. The full PIC system is used to simulate the generation of fast particles via laser plasma interactions. Data of the generated fast particles are copied to the reduced-field-solver PIC system in real time but retained in the full PIC system. The transport of the particles and energy deposition are calculated in the reduced-field-solver PIC system. In this way there is no longer any need to match the two field solutions as usually required in a single PIC system with a boundary to separate two field solvers. The resolution in both PIC systems in our approach can be taken as an usual one to satisfy the full field solver for certain maximum plasma density (e.g. $100 n_{c}$ ), since the reduced field solver need not a high resolution. We call this approach two-system PIC.

The outline of the paper is as follows. In Sec. II, a comparison is made about the noise between the full PIC simulation and the two-region PIC simulation, which also provides benchmark for the reduced field solver. In Sec. III, the basic algorithm for the two-system approach is presented. An example of integrated FI simulation with the two-system approach is given in Sec. IV. We conclude with a summary and discussion in Sec. V.

\section{COMPARISON BETWEEN THE REDUCED AND FULL FIELD SOLVERS}
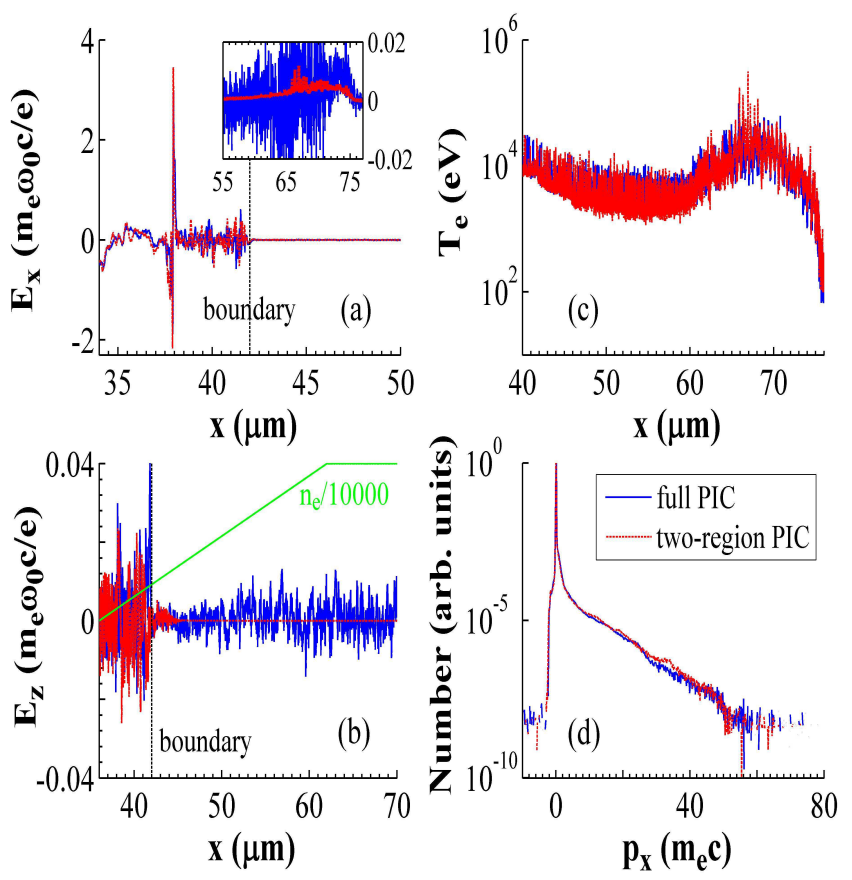

FIG. 1. Comparison of 2D simulation results obtained with the full PIC simulation (blue-solid lines) and the two-region PIC simulation (red-broken lines). (a)-(c) Spatial distributions of electric fields and electron temperature at the axis $(y=0)$. The inset in (a) is a close-up of the field. (d) Electron number distribution as a function of the longitudinal momenta. These results are given at $0.15 \mathrm{ps}$.

As mentioned in the introduction, our two-system PIC approach is based upon the two-region PIC model, where a reduced field solver is adopted for the high density region. The two-region PIC model has been benchmarked fully against the full PIC model in both $1 \mathrm{D}$ and $2 \mathrm{D}$ geometry in Ref. [26]. Here we present an extended benchmark in 2D geometry with a higher spatial resolution and a larger number of macroparticles to display the matching problem of the full and reduced field solvers on the boundary. In our simulations, the spatial resolution in both the $\mathrm{x}$ and $\mathrm{y}$ directions is $5 \mathrm{~nm}$. A cell is loaded with 400 macroparticles for each species. Figure 1 shows our benchmark simulation with our PIC code KLAPS (see the introduction of KLAPS in Appendix A) and illustrates the reduced field solver can be applied in high density plasma region, as shown in Ref. 26]. The simulation setup is as follows. The plasma density grows linearly from $0.2 n_{c}$ to $400 n_{c}$ between $x=36 \mu \mathrm{m}$ and $x=62 \mu \mathrm{m}$ and remains at a plateau of $400 n_{c}$ with a size of $14 \mu \mathrm{m}$. A laser pulse at wavelength $1 \mu \mathrm{m}$ propagates along the $+\mathrm{x}$ direction with peak intensity of $5 \times 10^{19} \mathrm{Wcm}^{-2}$, linear polarization along the $\mathrm{y}$ direction, and a full width at half maximum (FWHM) duration of 30 fs. To reduce 
the computation expense we take a planar laser profile so that the simulation box size along the y direction can be set small enough. In the two-region PIC simulation, the boundary at $x=42 \mu \mathrm{m}$ is taken, as illustrated in Figs. 1(a) and 1(c). The fields are given by the full field solver (solving the full Maxwell's equations as in a traditional PIC model [17, 19]) in the left region with the plasma density below $100 n_{c}$ and those are solved by the reduced Maxwell's equations 26] in the right region with the plasma density above $100 n_{c}$. In the full simulation there is no such a boundary and the fields in the whole simulation box are obtained by the full field solver. In both simulations, a Coulomb collision module and a 4th order current calculation are employed (see Appendix A) and the digital smoothing of fields around the boundary and high density region is taken as Ref. [26] (although there is no boundary in the full PIC simulation).

Here, the resolution and particle number per cell are taken to be much higher than those in usual simulations. However, the numerical noises of the fields in the full PIC simulation still appear to be much higher than those in the right region of the two-region PIC simulation, as clearly seen in Figs. 1(a) and 1(c). The noise of the former is 2-3 orders of magnitude higher than the latter, which will grow further if a lower resolution is taken. In particular, the noise of $E_{z}$ in the left region in the two-region PIC simulation is also rather higher than the one in the right region (note $E_{z}$ should vanish in theory since the laser polarization is along the y direction). One notices that the noise of the full field solution in the left region has spread to $x=46 \mu \mathrm{m}$ (the boundary at $x=42 \mu \mathrm{m})$ at this time. The value given by the reduced field solver is masked, which can result in the discontinuity of EM fields around the boundary of the two regions. This may limit the application of the two-region PIC approach if a long time's simulation is required (e.g., for FI simulation) and at the meanwhile a moderate resolution is employed due to limited computational resources.

\section{ALGORITHM FOR THE TWO-SYSTEM PIC APPROACH}

Our two-system PIC approach can completely avoid the problem of field discontinuities close to the boundary, even with a conventional resolution. This approach needs only a few modifications to the two-region PIC approach. We solve both the full and reduced Maxwell's equations in the whole simulation box independently in two systems. The two systems have their own respective macroparticles, which are independent of each other. We denote the full PIC system as system I and the reducedfield-solver PIC system as system II. In system I a traditional PIC algorithm with Coulomb collisions [24] is taken to simulate the generation of fast particles via laser plasma interactions. Because the ignition laser pulse cannot propagate into a region with high density plasma in a FI case, we lower artificially the density in this region to a given value when it exceeds this value (e.g., hundreds of $n_{c}$ ). Before the generated fast particles enter this region, their data (positions, momenta, masses, charges, weights, etc.) in system I are duplicated to system II in real time (and meanwhile these particles are retained in system I). In system II, the plasma density is taken as the real profile in the whole simulation box and the fields are solved with the reduced Maxwell's equations, similar to the ones used in the high density region in the tworegion PIC approach [26]. Note that the incident laser pulse is found only in system I. Obviously, there is no need to match the full and reduced field solutions in the two-system approach.

The duplication of the fast particle data is performed in the following way. In system I, one calculates the generation of fast particles and the transport to a region which is far enough away from interacting points of the laser. In the region, the data of the fast particles are copied to system II, where we call the region as the injection point of the fast particles. One can artificially reduce the plasma density from its real value behind the injection point to reduce numerical noise in system I, as mentioned above. In system II the plasma density is not changed artificially. The setup of the density and the injection point can be seen in Fig. 2 below as an example.

We present the algorithm equations of the field solver in system II (note that the full Maxwell's equations are taken in system I). The background currents of electrons and ions are calculated by the Ampere's law omitting the displacement current,

$$
\mathbf{J}_{b}=\frac{c}{4 \pi} \nabla \times \mathbf{B}-\mathbf{J}_{f},
$$

where $\mathbf{J}_{f}$ is the total current of fast electrons and ions, which is computed from the PIC particles directly. The background current calculated in this way avoids numerical noise in extremely high density, e.g., $300 \mathrm{~g} / \mathrm{cm}^{3}$. Faraday's law is used to advance the magnetic fields:

$$
\frac{\partial \mathbf{B}}{\partial t}=-c \nabla \times \mathbf{E} .
$$

The electric fields are solved by the Ohm's law:

$$
\mathbf{E}=\boldsymbol{\eta} \cdot \mathbf{J}_{b},
$$

where $\boldsymbol{\eta}$ is the classical resistivity [26, 27]. The field solver given by Eqs. (1)-(3) is the same with the one used in hybrid PIC model [20 23], the validation of which has been established. The largest difference between the hybrid PIC model and our system II is that the former considers the background plasma as a fluid denoting by $\mathbf{E}=\boldsymbol{\eta} \cdot \mathbf{J}_{b}$, whereas the latter takes the background as macroparticles as in a traditional PIC model. In principle, the calculation of the collisions between fast particles and the background plasma is more accurate in system II than that in hybrid PIC simulation since it uses a series of Monte Carlo tests between randomly-chosen particle pairs computed with the relativistic binary collision formula [24]. 
The main difference of the field solver by Eqs. (1)-(3) from the ones in the two-region PIC approach is that the latter retains the displacement current term in Eq. (1) to satisfy the numerical stability constraints [26]. Our simulation shows that such instability arises when the fluid quantities such as the electron density and electron average velocity are computed directly from the particles. This can be canceled in the following way even without the displacement current term. According to Eq. (1), one can easily obtain $\nabla \cdot \mathbf{J}=0$, where $\mathbf{J}=\mathbf{J}_{b}+\mathbf{J}_{f}$ is the total current. Applying the continuity equation $\nabla \cdot \mathbf{J}+$ $\partial \rho / \partial t=0$ of the fluid, one gets the charge conservation $\rho \equiv 0$ all the time if it meets initially. Here $\rho$ is the total charge density and it can be written by $\rho=e Z n_{i}-$ $e n_{e}-e n_{f}$, where $n_{e}$ and $n_{i}$ are densities of background electrons and ions and $n_{f}$ is the fast electron density. Hence, the background electron density can be given by $n_{e}=Z n_{i}-n_{f}$, which is consistent with the background current calculated by Eq. (1). In the case with high density plasma, $n_{e} \simeq Z n_{i}$ is a good approximation. In addition, the charge conservation is met automatically with the field solver with Eqs. (1)-(3) and one need not carry out the charge correction.

In the KLAPS code Eqs. (1)-(3) are computed as follows. Fast particle current $\mathbf{J}_{f}$ as well as $\mathbf{J}_{b}$ and the temperature of background electrons $T_{e}$ are defined at half-integer time points. Fields, densities of background electrons $n_{e}$, ions $n_{i}$, and fast electron density $n_{f, e}$, are defined at integer time points. Quantities $\mathbf{J}_{f}, T_{e}, n_{i}$, and $n_{f, e}$ are computed from the particles directly and the others are derived. Here $T_{e}, n_{e}$, and $n_{i}$ are used to calculate the classical resistivity. The half-integer and integer points in space are taken according to the traditional Yee scheme [19]. We first calculate the magnetic fields at time $(n+1 / 2) \triangle t$ using the quantities at $n \triangle t$ :

$$
\frac{\mathbf{B}^{n+1 / 2}-\mathbf{B}^{n}}{\triangle t / 2}=-c \nabla \times \mathbf{E}^{n} .
$$

Then compute the background current at $(n+1 / 2) \triangle t$ :

$$
\mathbf{J}_{b}^{n+1 / 2}=\frac{c \nabla \times \mathbf{B}^{n+1 / 2}}{4 \pi}-\mathbf{J}_{f}^{n+1 / 2} .
$$

Insert $\mathbf{J}_{b}^{n+1 / 2}$ into the Ohm's law and obtain:

$$
\mathbf{E}^{n+1 / 2}=\boldsymbol{\eta} \cdot \mathbf{J}_{b}^{n+1 / 2} .
$$

By use of

$$
\mathbf{E}^{n+1}=2 \mathbf{E}^{n+1 / 2}-\mathbf{E}^{n}
$$

one gets $\mathbf{E}^{n+1}$. Finally, calculate the magnetic fields at $(n+1) \triangle t$ using those at $(n+1 / 2) \triangle t$ :

$$
\frac{\mathbf{B}^{n+1}-\mathbf{B}^{n+1 / 2}}{\triangle t / 2}=-c \nabla \times \mathbf{E}^{n+1} \text {. }
$$

In system II digital smoothing is taken for all fields, currents, temperatures, and densities. We examine two spatial smoothing algorithms, one is

$$
F_{i}=\frac{F_{i-1}+2 F_{i}+F_{i+1}}{4}
$$

and the other is

$$
F_{i}=\frac{F_{i-2}+2 F_{i-1}+3 F_{i}+2 F_{i+1}+F_{i+2}}{9},
$$

the subscript $i$ indicates the spatial grid index in either $x$ or $y$ direction, e.g., for 2D case. This digital smoothing is performed in both $x$ and $y$ directions. The The two algorithms give nearly the same results. Considering that 4th-order "zigzag" algorithm is employed in both systems (see the introduction of KLAPS in Appendix A), we take the latter to match 4th-order interpolation used in both particle pusher and current calculation in KLAPS.

\section{AN EXAMPLE OF TWO-SYSTEM PIC SIMULATION}

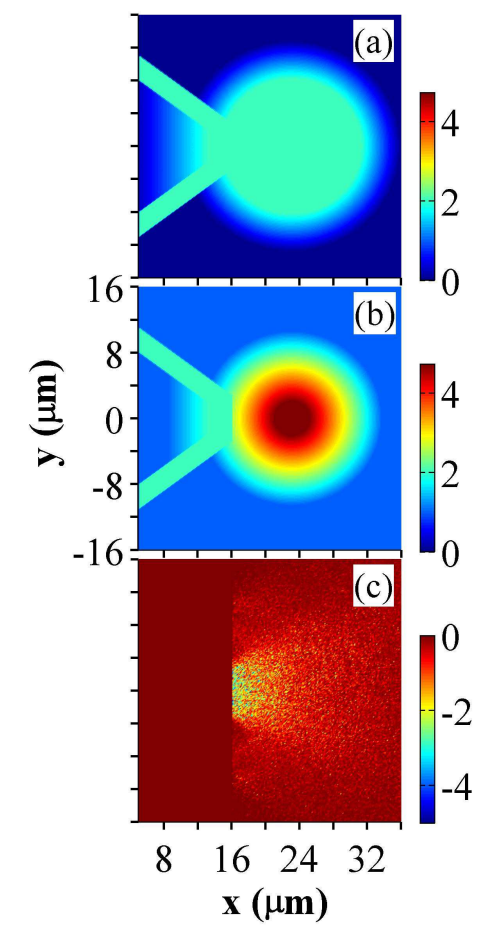

FIG. 2. Initial plasma electron densities $\lg \left(n_{e} / n_{c}\right)$ taken in system I (a) and system II (b). (c) The fast particle current $J_{f, x} / e n_{c} c$ in system II at 0.2 ps. The injection point is taken at $x=16 \mu \mathrm{m}$.

In Figs. 2(a) and 2(b), we demonstrate the density setup in the two systems through an example of FI. A compressed target is taken with the uniform density of $300 \mathrm{~g} / \mathrm{cm}^{3}$ (or $54000 n_{c}$ ) at the core area within a circle of the radius $2 \mu \mathrm{m}$ and the surrounding density decreasing exponentially with a scalelength $1 \mu \mathrm{m}$ along the radial direction away from the core center. A cone is embed into the target with the cone angle of 45 degree, the wall depth of $3 \mu \mathrm{m}$, the density of $100 n_{c}$, tip size of $4 \mu \mathrm{m}$, and the inner length of $8 \mu \mathrm{m}$. Inside the cone a preplasma has an exponential profile with a scalelength $2 \mu \mathrm{m}$ along 
the $\mathrm{x}$ direction and is distributed uniformly in the $\mathrm{y}$ direction. System II takes this plasma density profile with a pedestal of $10 n_{c}$, as shown in Fig. 2(a). In system I the density profile is changed in such a way as when the plasma density is above the cone wall density $100 n_{c}$, it is lowered to be $100 n_{c}$, as displayed in Fig. 2(b). The injection point of the fast particles is chosen at the cone tip end with $x=16 \mu \mathrm{m}$. To be related with FI studies, we define such particles to be fast that the energy is higher than $0.1 \mathrm{MeV}$ and the forward momentum $p_{x}>0.45 m_{e} c$ $(50 \mathrm{keV})$ for electrons or $p_{x}>46 m_{e} c(0.1 \mathrm{MeV})$ for tritium ions. The data of such fast particles are copied from system I to II in real time.

The two systems have the same simulation box size of $48 \mu \mathrm{m} \times 48 \mu \mathrm{m}$ in $x \times y$ directions. The resolutions in both the $\mathrm{x}$ and $\mathrm{y}$ directions are $(1 / 32) \mu \mathrm{m}=1.96 \mathrm{c} / \omega_{\mathrm{p}}$ $\left(\omega_{p}^{2}=100 n_{c} \times 4 \pi e^{2} / m_{e}\right)$ and the temporal resolution is $0.067 \mathrm{fs}=1.25 / \omega_{p}$. Initially, 25 electrons and tritium ions cover a cell both in system I and II. The initial temperatures of electrons and ions are uniformly in space, which is $1 \mathrm{keV}$. A laser pulse with wavelength of $1 \mu \mathrm{m}$ propagates along the $+\mathrm{x}$ direction. It is linearly polarized along the y direction with the electric fields $E=a_{0} \exp \left(-y^{2} / r_{0}^{2}\right) f(\xi) \sin (2 \pi \xi)$, where $a_{0}=12.1$ corresponding to $2 \times 10^{20} \mathrm{~W} / \mathrm{cm}^{2}, \xi=t-x / c, r_{0}=4 \mu \mathrm{m}$, the temporal profile $f(\xi)$ is taken as a trapezoid, i.e., a plateau of 1 ps between $3.33 \mathrm{fs}$ rising and decreasing regions. The simulation time is $1.2 \mathrm{ps}$. Here, these resolutions and particle number per cell have been examined in detail as found in Appendix B.

Figure 2(c) shows the longitudinal fast current in system II at 0.2 ps. This fast current is contributed not only by the fast particles injected from system I but also by the particles originating in system II which gain high enough energy from the fast particle influx. We calculate the fast current from the electrons with energy $E>5 T_{e}(x, y)$ and $E>50 \mathrm{keV}$ as well as the tritium ions with $E>5 T_{i}(x, y)$ and $E>50 \mathrm{keV}$, where $T_{e}(x, y)$ and $T_{i}(x, y)$ are the local temperatures of electrons and ions.

We first check energy conservation of the two systems. Absorbing boundary conditions for both particles and EM fields are taken in the two systems and energy of the particles and fields fled away from the simulation box is recorded. Figure 3(a) displays the energy gain of system I normalized by the incident laser energy. This figure illustrates that the energy conservation remains good in system I. The highest error value in this system is $3.3 \%$ at 1.2 ps. Figure 3(b) shows the energy of system II which includes the residual energy of the fast particles, the gain of background particles, and the EM fields computed by Eqs. (4)-(8). The energy is normalized by the total energy of the fast particles injected at different times. The curve of the energy evolution begins at 46 fs when some fast electrons start to be injected. It shows good energy conservation within the whole simulation period of 1.2 ps, where the conservation is kept within $0.4 \%$. Note that the error for energy conservation is larger for system I, which is due to the higher noise of the full field
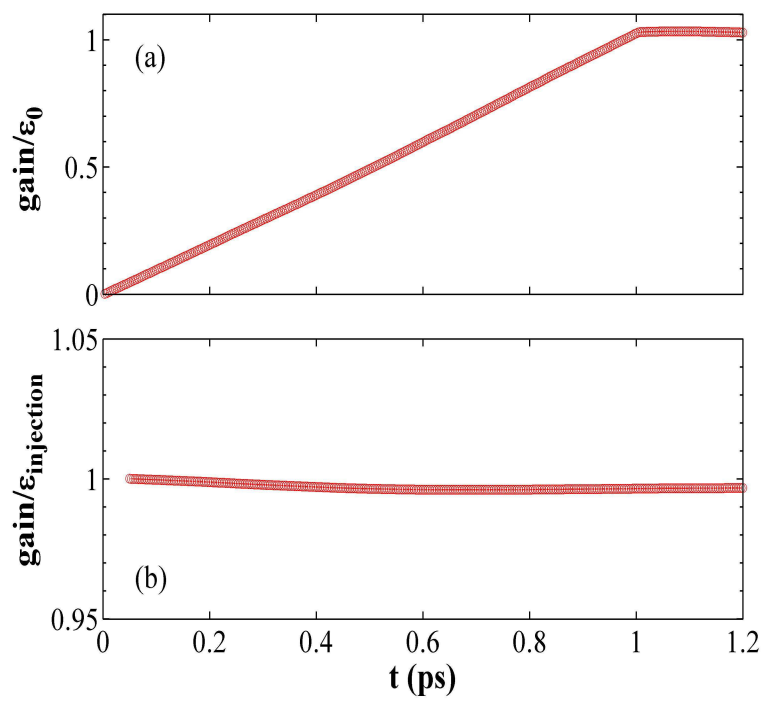

FIG. 3. (a) Total energy change with time in system I, which is normalized by the incident laser energy $\varepsilon_{0}$. (b) Total energy change of system II normalized by the total energy of the fast particles injected $\varepsilon_{\text {injection }}$ at different times.

solver. This noise can be lowered by increasing the number of macroparticles in system I. A benchmark of our two-system PIC model against the full PIC model is also presented in Appendix D.

In the following Figs. 4-8 we plot spatial distributions of the EM fields, currents, and temperatures in system II at different times. Figure 4 shows the fast currents and longitudinal electric fields at $0.2,0.6$, and $1 \mathrm{ps}$. Compared to the fast current with the peak at the injection point in Fig. 4(a) at earlier time, Figs. 4(c) and 4(e) display the peaks shift to the region between the injection point and the core area beginning at $x=21 \mu \mathrm{m}$ because a large number of fast electrons are slowed down by strong collisions and accumulated at this region. Except with very high energy, fast electrons are mostly barred from the core and loop around the core surface. The fast current appears the minimum at the core and the area just behind it. A similar pattern for the $E_{x}$ distribution can be found in Figs. 4(b), 4(d), and 4(f) since $E_{x}=\eta_{x} J_{b, x} \simeq-\eta_{x} J_{f, x}$. A clearer picture can be seen in Fig. 5. Compared Figs. 5(a) and 5(b), one can observe that the electric field attenuates with the time although the current is enhanced. The reason is that $\eta$ strongly depends on the local electron temperature with $\eta \propto T_{e}^{-3 / 2}$. The temperature grows with time and therefore the electric field attenuates.

The distributions of the total currents (sum of the fast and background currents) and magnetic fields are illustrated in Fig. 6. Fine filamentary structures can be seen in the current all the time. It is also seen that the total currents appear positive sign at the core area because the return current exceeds the local $J_{f, x}$. The total current is negative in the surrounding area with lower densities, where $J_{f, x}$ is not completely neutralized by $J_{b, x}$. There 

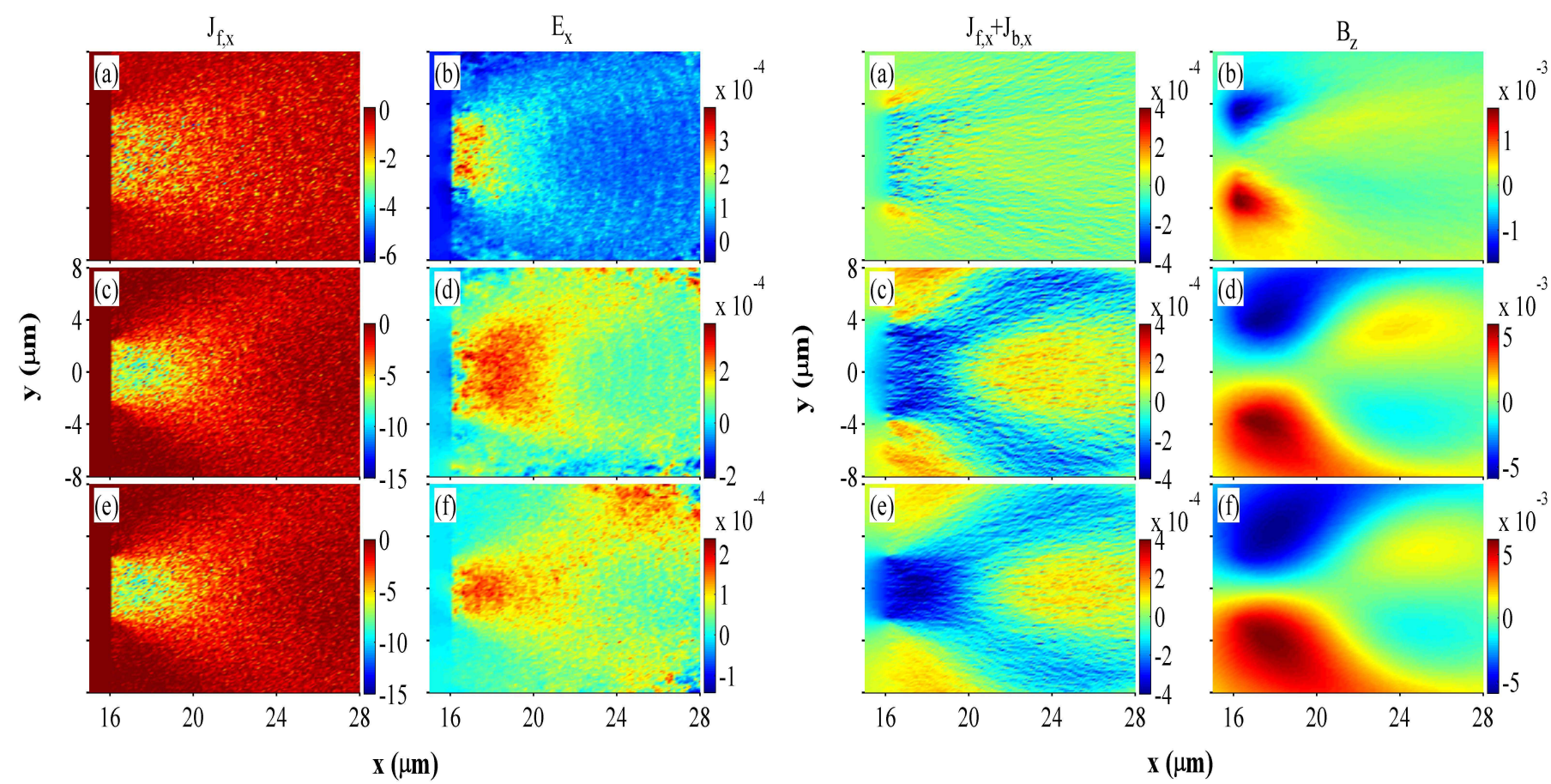

FIG. 4. Snapshots of spatial distributions of the fast current $J_{f, x}$ (the left column) and $E_{x}$ (the right column), which are normalized by $e n_{c} c$ and $m_{e} \omega_{0} c / e$, respectively. The three rows correspond to $0.2 \mathrm{ps}, 0.6 \mathrm{ps}$, and $1 \mathrm{ps}$.

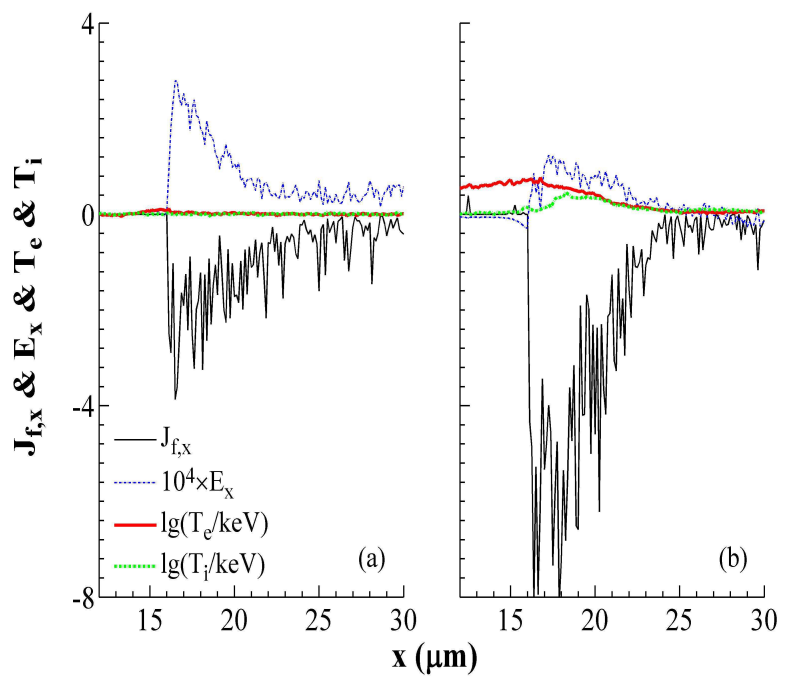

FIG. 5. Spatial distributions of $J_{f, x} / e n_{c} c, 10^{4} \times e E_{x} / m_{e} \omega_{0} c$, the background temperatures of electrons $T_{e}$ and ions $T_{i}$ on the axis $(\mathrm{y}=0)$ at $0.2 \mathrm{ps}(\mathrm{a})$ and $1.2 \mathrm{ps}(\mathrm{b})$.

are also positive total currents in the low density area just adjacent to and outside the fast current peak area (see Fig. 4), which are excited by the adjacent fast ones. Accordingly, the magnetic fields show two groups of peaks with opposite signs: one around the core and the other around the injection point.

FIG. 6. Snapshots of spatial distributions of the total current along the $\mathrm{x}$ direction (the left column) and $B_{z}$ (the right column), which are normalized by $e n_{c} c$ and $m_{e} \omega_{0} c / e$, respectively. The three rows correspond to $0.2 \mathrm{ps}, 0.6 \mathrm{ps}$, and 1 ps.

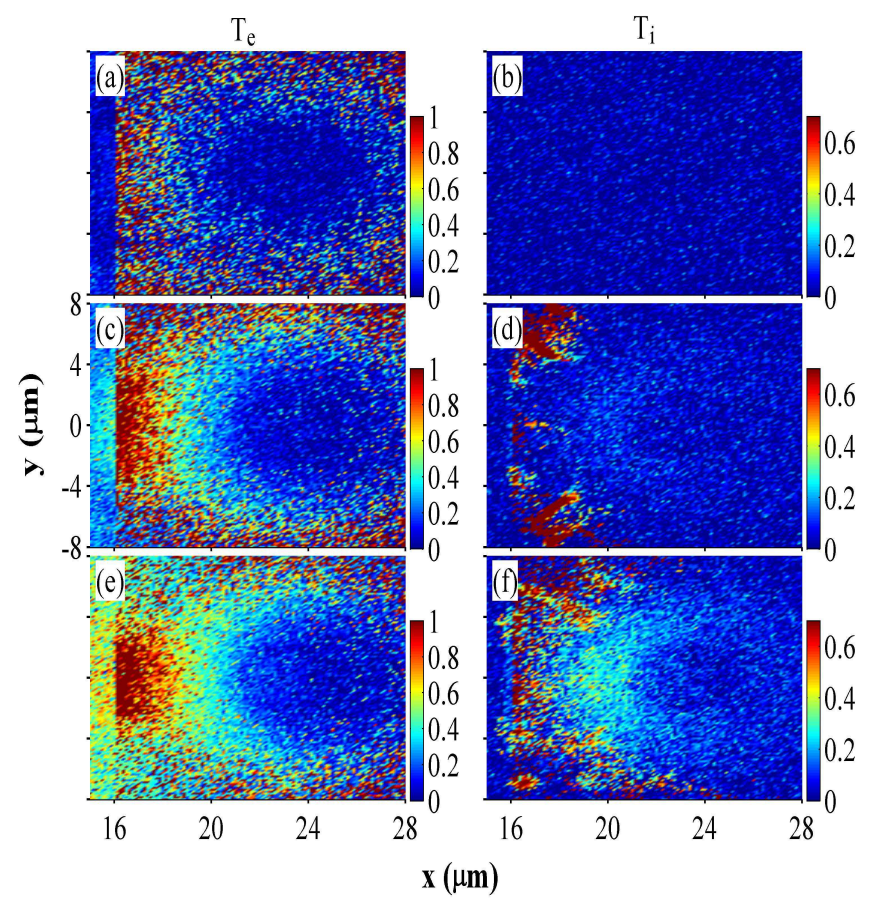

FIG. 7. Snapshots of spatial distributions of $\lg \left(T_{e} / \mathrm{keV}\right)$ (the left column) and $\lg \left(T_{i} / \mathrm{keV}\right)$ (the right column), where $T_{e}$ and $T_{i}$ are temperatures of all the electrons and ions. The three rows correspond to $0.2 \mathrm{ps}, 0.6 \mathrm{ps}$, and $1 \mathrm{ps}$. 

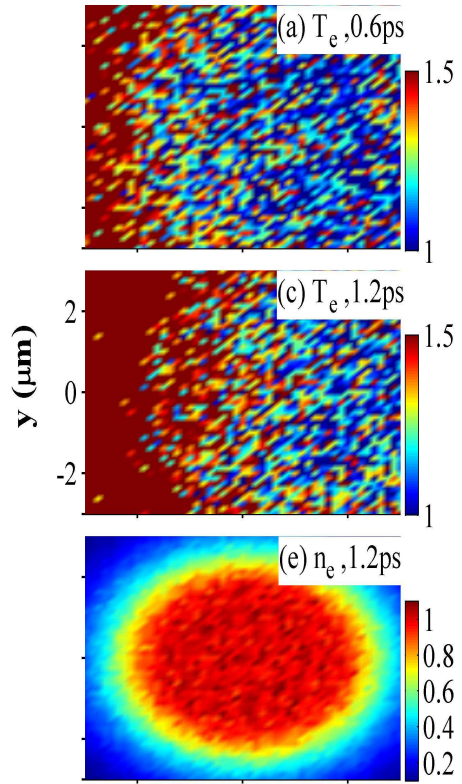

21
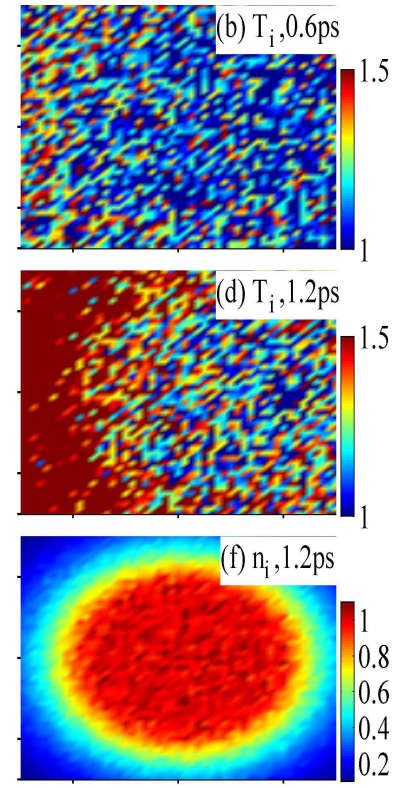

23

25

$\mathbf{x}(\mu \mathrm{m})$

FIG. 8. Snapshots of spatial distributions of $\lg \left(T_{e} / \mathrm{keV}\right)[(\mathrm{a})$ and (c)] and $\lg \left(T_{i} / k e V\right)$ [(b) and (d)], where (a), (b) correspond to $0.6 \mathrm{ps}$ and (c), (d) to $1.2 \mathrm{ps}$, and $T_{e}$ and $T_{i}$ are temperatures of all the electrons and ions. Spatial distributions of densities of the electrons and ions around the core at $1.2 \mathrm{ps}$ are given in (e) and (f), respectively, which are computed from the particles directly and normalized by 54000 $n_{c}$.

The temperatures of electrons and ions at different times are plotted in Fig. 7 (the values of the temperatures are shown within a limited range). One sees that the electron temperature increases quickly in the area surrounding the core as the fast electrons arrive (see Fig. 4). The core always remains colder however: between the core and the injection point a temperature front is observed to advance slowly towards the core. After some delay, the ion temperature also increases up to $T_{e}$ due to collisions. The temperature peaks in Fig. $7(\mathrm{~d})$ appear at low density regions where the ions are easy to heat. A clearer picture in Fig. 5 can be seen that the ion temperature trails with the electron temperature, which grows with time. One also observes that the electron temperature at the region in front of the injection point is enhanced at latter time because some electrons acquire velocities along the $-\mathrm{x}$ direction via collisions and some of these go to the region.

A zoom of the temperatures around the core is given in Figs. 8 (a)- $8(\mathrm{~d})$. One sees a slow process of the electron heating towards the core. The ion heating with a delay is also observed. One notice that there is inhomogeneity in the electron and ion temperatures even at the core with an extremely high density. With such a low temperature, the rate eliminating the inhomogeneity does not depend

on the collision frequency but the speed of the particles. Figures 8(e) and 8(f) display the densities of the electrons and ions around the core at $1.2 \mathrm{ps}$. Deviation of the densities from the initial values is not large.

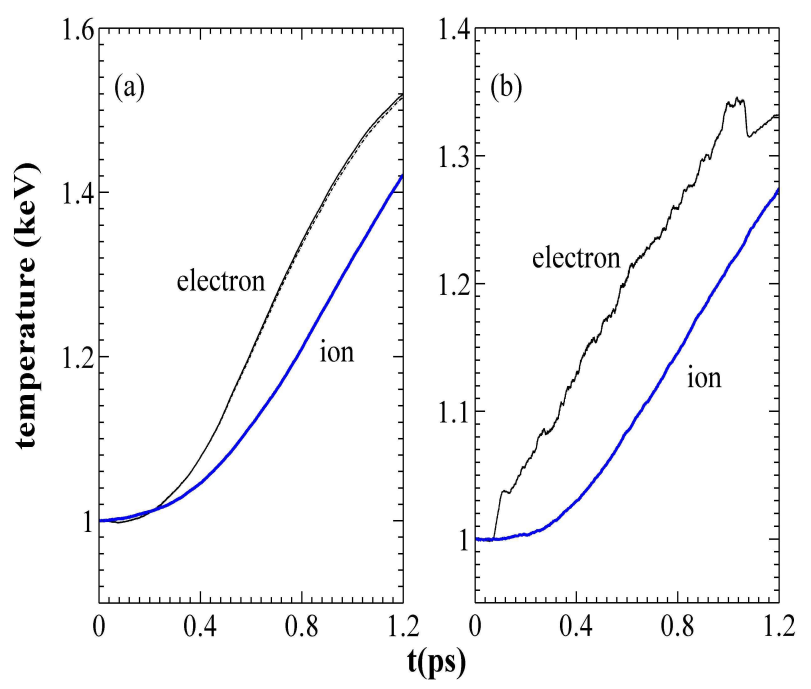

FIG. 9. (a) Evolution of the temperatures of the electrons and ions of system II, where the broken lines denote the gain from collision and the solid lines are for the total gain. (b) Evolution of the temperatures of the electrons and ions within the core area.

It is interesting to present the temporal evolution of the temperatures at the core area in Fig. 9(b). The temperature of the electrons at the core (including fast electrons just arrived) are always higher than $T_{i}$, which leads to the continuous heating to the ions. The ions are heated to about $1.28 \mathrm{keV}$ at $1.2 \mathrm{ps}$. Figure 9(a) illustrates the evolution of the temperatures of the electrons and ions of system II (not including the fast particles injected). The broken lines mean the gain from collision only while the solid lines correspond to the total gain. It is shown that the collisional heating dominates the resistive heating nearly completely. Note that the latter will be enhanced provided the scalelength of the density surrounding the core grows, in which the ratio of plasma with lower density goes up.

The bars in Fig. 10(a) show the ratios of the total energy of fast particles and fields to the incident laser energy at 1.2 ps. The reflected light carries off $25 \%$ energy of the incident laser. The conversion efficiency to fast particles is $46 \%$, though $87 \%$ of these leave the simulation box because of large divergence as well as too high energy. Note that $0.47 \%$ energy is absorbed by fast ions. Figure 10(b) presents the energy gain partition of system II. $4.6 \%$ energy of the incident laser is transferred to the background plasma and the conversion efficiency to the core is only $1.2 \%$. These values should grow slightly if a longer simulation is taken since about $1.5 \%$ laser energy is covered by the fast particles which are still located in the system. It should be pointed out that we have chosen 


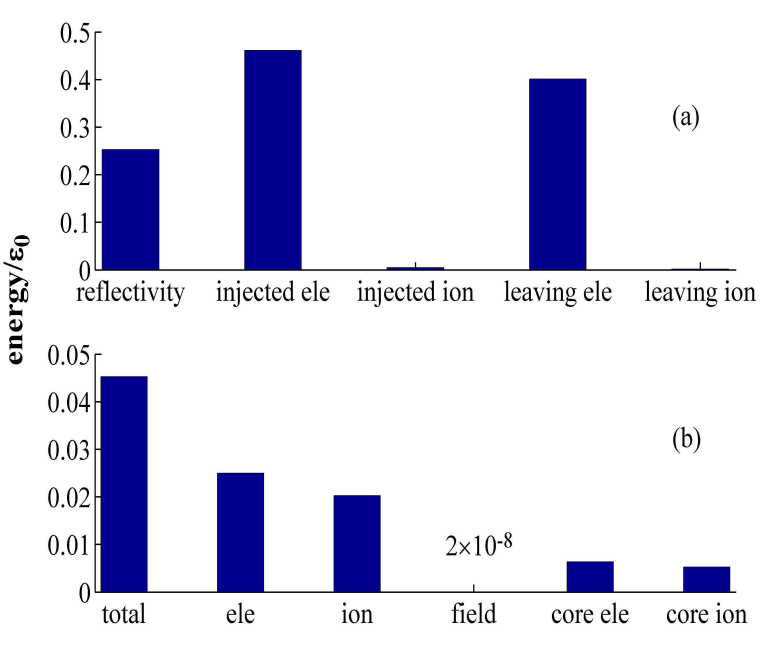

FIG. 10. (a) Energy of reflected light, the fast electrons and ions injected to system II, and the fast electrons and ions leaving the simulation box, respectively, normalized by the incident laser energy $\varepsilon_{0}$. (b) Energy gain of system II, background electrons and ions, fields, and the electrons and ions at the core area, respectively, normalized by $\varepsilon_{0}$. These values are obtained at $1.2 \mathrm{ps}$.

too high laser intensity to examine our approach with a powerful fast current. The optimized laser parameters for ignition should be investigated further in the future.

\section{SUMMARY AND DISCUSSION}

In summary, we have proposed a two-system PIC approach with which integrated simulation can be performed including fast particle generation via laser plasma interaction and fast particle transport and energy deposition in extremely high density plasma. This approach can be applied to an integrated simulation of fast ignition with real target density, e.g., $300 \mathrm{~g} / \mathrm{cm}^{3}$, which has been illustrated by the example in this paper. Also, it may be used to simulate the transport of fast particles in solid targets with a self-consistent calculation of the fast particle generation, e.g., in the context of hard x-ray sources. To apply this approach, a Coulomb collision module as well as a high order current scheme should be included in the PIC code.

In the two-system approach, a full PIC model with collisions is taken in the first system, which is used to calculate the fast particle generation. In the second system a reduced field solver is employed, as used in hybrid PIC approach. This system computes fast particle transport and energy deposition in the high density plasma. The plasmas in both systems are modeled by macroparticles as in a conventional PIC code. If the energies of particles generated in the first system are above some threshold (e.g., hundreds of $\mathrm{keV}$ adjustable according to different physical problems), their data will be copied to the second system. The fast particles include not only electrons but also ions and therefore, the two-system PIC model could also be applied in ion transport and ion fast ignition.

In the current version, we have taken the same simulation box and resolutions in the two systems for simplicity. In principle, one can reduce the simulation box size in the full PIC system if there is a large enough space between the injection point of the fast particles and the right boundary of the simulation box, as shown in Appendix C. Also, the two systems may have different resolutions in time and space. For example, the resolutions in the second system can be taken to be lower than the first system to save the computation expense.

Basically, in the two-system model the hot particle transport is simulated via a relay channel between the two PIC systems. The relay point between the two systems is the injection point of the fast particles. To assure the validation of the two-system model, the relay point should be taken such that: it is far away from the laser interaction zone; the plasma density at this point should be sufficiently high enough to satisfy the fluid approximation applied in the reduced field solver; the density should also be low enough to reduce the noise in the full PIC system. In this paper we have used a relay point with a density of $100 n_{c}$. With a longer duration, e.g., $10 \mathrm{ps}$, the laser pulse can enter into a deeper region with a higher plasma density via the hole boring effect. The relay point need be taken with an even higher density. This is the case for FI with a cone-free target as used in our recent work 28], in which the two-system approach has been applied to study a new form of magnetically assisted FI.

\section{ACKNOWLEDGMENTS}

W.M.W. acknowledges support from the Alexander von Humboldt Foundation. This work was supported by the National Basic Research Program of China (Grants No. 2013CBA01500) and NSFC (Grants No. 11375261, No. 11105217, No. 11129503, No. 113111048, No. 11375262 , and No. 11135012). The authors gratefully acknowledge the computing time granted by the JARA-HPC and VSR committees on the supercomputer JUQUEEN at Forschungszentrum Jülich.

\section{Appendix A: On the PIC code KLAPS}

The PIC code KLAPS (kinetic laser plasma simulation) has been developed by Wei-Min Wang and ZhengMing Sheng from the 2D serial, basic version of KLAP [29] to a parallel code with both $2 \mathrm{D}$ and $3 \mathrm{D}$ versions. Besides the basic properties of a traditional PIC code [1719], KLAPS also includes field ionization, Coulomb collision, radiation reaction, first-fourth order "zigzag" cur- 
rent calculation, a Maxwell-equation-reduced field solver, a dispersion-free field solver, moving window technology, and absorbing and/or periodic boundary conditions in any direction. In this paper, Coulomb collision, fourth order "zigzag" current calculation, absorbing boundary conditions are applied in the simulations.

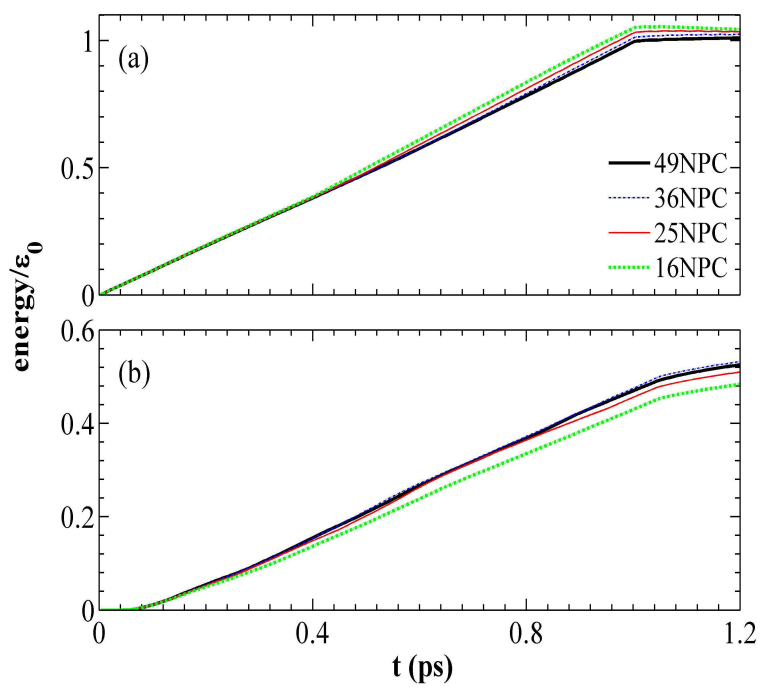

FIG. 11. (a) Evolution of the total energy gain of system I with different particle numbers per cell (NPC) and (b) the corresponding fast particle energy injected into system II, where the energy is normalized by the total energy of the incident laser pulse.

The Coulomb collision module has been developed based on the scheme proposed by Sentoku and Kemp [24]. This scheme computes electron-electron, electron-ion and ion-ion collisions with a relativistic formula of two-body collision [24, 30]. Through a series of two-body collision tests of randomly chosen pairs in a cell [31], it can simulate many-body collision and it can perfectly keep the conservation of both energy and momentum per time step with macroparticles of different weights 24]. KLAPS calculate currents by 1st-4th order "zigzag" scheme. The first order algorithm proposed by Umeda et al. [32] in 2003, which shows higher efficiency than the one proposed by Esirkepov 33]. We extended it to 2nd-4th order with charge conservation [34, 35] and implemented them in KLAPS. The field ionization of atoms is calculated according to the Ammosov-Delone-Krainov (ADK) formula [36, 37]. Besides tunneling ionization at high laser intensities with the Keldysh parameter $\gamma_{K}<1$, the ADK model can also be applied in the intermediate regime between multiphoton ionization and tunneling with $1<\gamma_{K}<8$ at low laser intensities [38], where the Keldysh parameter is refined by $\gamma_{K}=\sqrt{\Phi / 2 U_{p}}[39]$, $\Phi$ is the ionization potential, and $U_{p}$ is the ponderomotive potential of the laser pulses. With this module it is possible to simulate ionization at a wide range of laser intensities, e.g., above $10^{13} \mathrm{~W} / \mathrm{cm}^{2}$. We have applied this module to investigate terahertz $(\mathrm{THz})$ radiation genera- tion from gas ionization [40 43]. The radiation reaction effect is added in the particle motion equation, where the radiation damping force is calculated according to the Landau-Lifshitz formula [44]. With this module the code has the potential to simulate laser plasma interactions with the intensity up to $10^{22} \mathrm{~W} / \mathrm{cm}^{2}$, above which weak quantum electrodynamics effects starts to work.

\section{Appendix B: Requirement of resolutions}

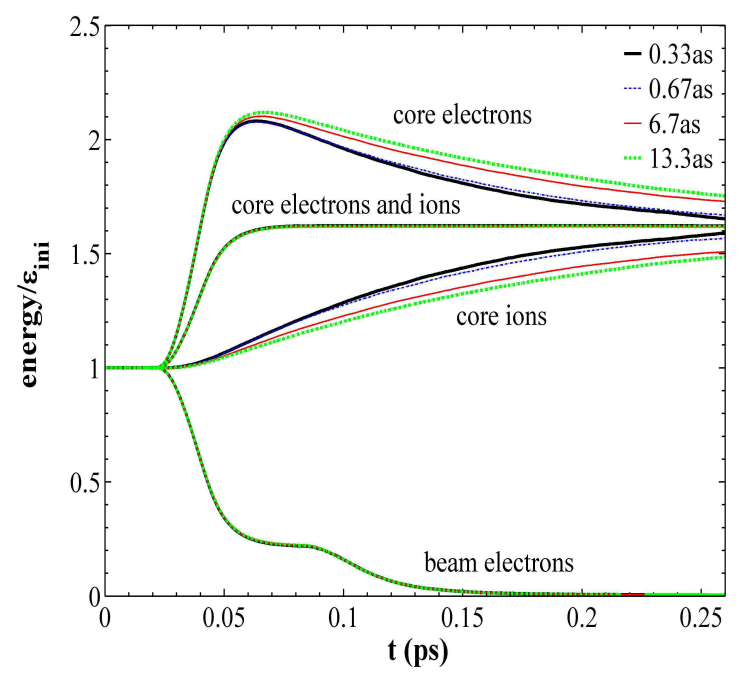

FIG. 12. Evolution of the average energy of the core electrons and ions as well as fast electrons with different collision time steps, where the energy units are taken as the initial values $\varepsilon_{\text {ini }}$ of each species.

We present simulations to examine the requirement of resolutions in the two systems, respectively. The density setup in the two systems is taken the same as shown in Fig. 2. The cone angle and tip size is changed to 20.6 degree and $2 \mu \mathrm{m}$. The same laser parameters are employed except a smaller spot radius of $r_{0}=2 \mu \mathrm{m}$. The simulation size $40 \mu \mathrm{m} \times 32 \mu \mathrm{m}$ is taken in $x \times y$ directions.

The requirements of the first system are mainly limited by the numerical noise caused by unresolved plasma oscillation which is characterized by the plasma frequency $\omega_{p}$ and skin depth $c / \omega_{p}$. Hence, one usually need take the spatial cell size $\Delta x$ and the temporal step $\Delta t$ to be comparable with or smaller than $c / \omega_{p}$ and $1 / \omega_{p}$, respectively. These restraints can be relaxed with a high order interpolation. We take the cone wall density of 100 $n_{c}$ and the highest density is also this value in system I. By use of the 4th order "zigzag" scheme it is shown that the noise can be controlled well with a spatial size $\Delta x=\Delta y=(1 / 32) \mu m=1.96 c / \omega_{p}$ and the time step $\Delta t=0.64 \Delta x / c=1.25 / \omega_{p}$ if the particle number per cell (NPC) is larger than 16, as shown in Fig. 11(a). This figure illustrates the evolution of the system energy normalized by the total energy of the incident laser pulse. 
At 1.2 ps the errors caused by the noise are $0.94 \%, 2.3 \%$, $3.2 \%, 4.3 \%$, respectively, for $49 \mathrm{NPC}, 36 \mathrm{NPC}, 25 \mathrm{NPC}$, and 16 NPC.

Figure 11 (b) plots the fast particle energy injected to system II. We change NPC to check the anomalous macroparticle stopping effect, which limits the highest number of real particles denoting by a macroparticle or the weight of a macroparticle. The evolution curve with $36 \mathrm{NPC}$ is nearly coincident with the one with $49 \mathrm{NPC}$ and its error ratio to the latter is $1.3 \%$ at $1.2 \mathrm{ps}$. The errors are $4.3 \%$ and $9.2 \%$ for the cases with $25 \mathrm{NPC}$ and 16 NPC. Summing up the two examination results given in Figs. 11(a) and 11(b), $25 \mathrm{NPC}$ is good enough to controlled both the noise and the anomalous stopping effect for system I.

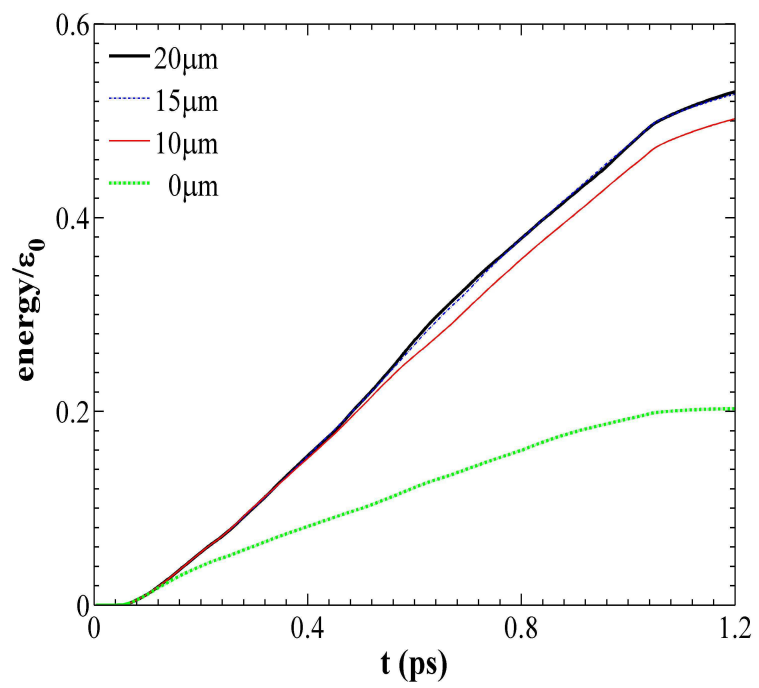

FIG. 13. Fast particle energy injected into system II, where it is normalized by the total energy of the incident laser $\varepsilon_{0}$. Different curves correspond to the data of the fast particles deleted at $0 \mu \mathrm{m}, 10 \mu \mathrm{m}, 15 \mu \mathrm{m}, 20 \mu \mathrm{m}$ away from the injection point in system I.

In system II the reduced field solver is applied which has much lower resolution requirement than the full solver since the fast-varied displacement current is omitted. Simply, one can safely take the same resolutions as system I. The bottleneck of the temporal resolution is imposed by collisions. One need make sure that the time step of the collision $\Delta t_{c}$ is smaller than the collision period $\tau_{c}=1 / \nu_{c}$ (the principle to choose $\Delta t_{c}$ can be found in Refs. [24, 30]), where $\nu_{c}=4 \pi e^{2} n_{e} L /\left(p_{\text {rel }}^{2} v_{r e l}\right)$ is the relativistic, two-body collision frequency of a particle pair [24], $L$ is the Coulomb logarithm, as well as $p_{\text {rel }}$ and $v_{r e l}$ the relative momentum and velocity of a particle in the rest reference of the other. We evaluate $\tau_{c}$ with the core density of $300 \mathrm{~g} / \mathrm{cm}^{3}=54000 n_{c}$ and temperature of 1 $\mathrm{keV}$. For simplicity we compute $p_{\text {rel }}$ with the assumption of the core particle at rest. For the collision between the core electron and fast electrons of $1 \mathrm{MeV}, \tau_{c}=424$ fs. This value is $19 \mathrm{fs}, 0.93 \mathrm{fs}$, respectively, for fast electrons of $0.1 \mathrm{MeV}, 0.01 \mathrm{MeV}$. The period of collision between a core electron and ion is much smaller, which is 59 as with the initial temperature $1 \mathrm{keV}$. We take a small enough $\Delta t_{c}=0.33$ as to resolve all the collision periods as the standard result. We also take $\Delta t_{c}$ between 0.67 as and 13.3 as to compare with the standard result. To only check the collision effect, we take all of the electrons in the cone with monoenergetic energy of $0.3 \mathrm{MeV}$ and the motion along $+\mathrm{x}$ direction and no laser pulse incident. One can see in Fig. 12 that the evolution of the fast electrons as well as the whole core electrons and ions nearly coincide when $\Delta t_{c}$ is changed, since all the $\Delta t_{c}$ used are much smaller than the collision period between the fast electrons and the core. The gain of the core ion temperature is $0.6 \mathrm{keV}$ with $\Delta t_{c}=0.33$ as at the time of 0.27 ps. From the value, the deviation of the gain with $\Delta t_{c}=0.67$ as, 6.7 as, and 13.3 as, respectively, is $3 \%$, $13 \%$ and $16 \%$. Therefore, $\Delta t_{c}=0.67$ as is good enough. Considering that the collision period will increase with the enhancing core temperatures, one could use a larger $\Delta t_{c}$. If one focuses on the energy transfer from the fast electrons to the core, $\Delta t_{c}$ can be taken to be much larger, e.g., $\Delta t_{c}=13.3$ as.

\section{Appendix C: Comparison between particle data duplication and migration}

In all of the previous simulations, we have copied fast particle data from system I to II and meanwhile these fast particles still exist in system I. If the data are deleted from system I just after transferred to system II, the computation expense can be saved. However, an unphysical electric field will be formed around the injection point in system I to block the coming particles. This effect on the particle injection is shown in Fig. 13. If the fast particle data deleted in system I immediately at the injection point after they transferred, the injection energy is reduced considerably. However, if the fast particle data deleted far away from the injection point, i.e., above 15 $\mu m$, the injection electron energy is affected slightly.

\section{Appendix D: Benchmark of two-system PIC}

Figure 14 shows the benchmark of the two-system PIC model against the full and two-boundary PIC models, where the results of the full and two-boundary PIC simulations have been presented in Fig. 1. Basically the field solver used in the second system of our two-system PIC model is nearly the same as the one taken in the high density region in the two-region PIC, as addressed in Sec. III. Therefore, the two simulation results show good agreement as seen in Fig. 14. Here, we adopt a decreased spatial resolution of $20 \mathrm{~nm}$ in the two-system simulation while it is $5 \mathrm{~nm}$ in the other two simulations. 
[1] M. Tabak, J. Hammer, M. Glinsky, W. Kruer, S. Wilks, J. Woodworth, E. Campbell, M. Perry, and R. Mason, Phys. Plasmas 1, 1628 (1994).

[2] R. Kodama, P. A. Norreys, K. Mima, A. E. Dangor, R. G. Evans, H. Fujita, Y. Kitagawa, K. Krushelnick, T. Miyakoshi, N. Miyanaga, T. Norimatsu, S. J. Rose, T. Shozaki, K. Shigemori, A. Sunahara, M. Tampo, K. A. Tanakaka, Y. Toyama, T. Yamanaka, and M. Zepf, Nature (London) 412, 798 (2001).

[3] Z.-M. Sheng, Y. Sentoku, K. Mima, J. Zhang, W. Yu, and J. Meyer-ter-Vehn, Phys. Rev. Lett. 85, 5340 (2000).

[4] Z. L. Chen, R. Kodama, M. Nakatsutsumi, H. Nakamura, M. Tampo, K. A. Tanaka, Y. Toyama, T. Tsutsumi, and T. Yabuuchi, Phys. Rev. E 71, 036403 (2005).

[5] A. L. Lei, K. A. Tanaka, R. Kodama, G. R. Kumar, K. Nagai, T. Norimatsu, T. Yabuuchi, and K. Mima, Phys. Rev. Lett. 96, 255006 (2006).

[6] Y. T. Li, X. H. Yuan, M. H. Xu, Z. Y. Zheng, Z. M. Sheng, M. Chen, Y. Y. Ma, W. X. Liang, Q. Z. Yu, Y. Zhang, F. Liu, Z. H. Wang, Z. Y. Wei, W. Zhao, Z. Jin, and J. Zhang, Phys. Rev. Lett. 96, 165003 (2006).

[7] A. Debayle, J. J. Honrubia, E. d Humieres, and V. T. Tikhonchuk, Phys. Rev. E 82, 036405 (2010).

[8] Y.-Q. Cui, W.-M. Wang, Z.-M. Sheng, Y.-T. Li, and J. Zhang, Plasma Phys. Contr. Fusion 55, 085008 (2013).

[9] J. J. Santos, F. Amiranoff, S. D. Baton, L. Gremillet, M. Koenig, E. Martinolli, M. Rabec Le Gloahec, C. Rousseaux, D. Batani, A. Bernardinello, G. Greison, and T. Hall, Phys. Rev. Lett. 89, 025001 (2002).

[10] Toshikazu Matsumoto,Toshihiro Taguchi, and Kunioki Mima, Phys. Plasmas 13, 052701 (2006).

[11] Y. T. Li, M. H. Xu, X. H. Yuan, W. M. Wang, M. Chen, Z. Y. Zheng, Z. M. Sheng, Q. Z. Yu, Y. Zhang, F. Liu, Z. Jin, Z. H. Wang, Z. Y. Wei, W. Zhao, and J. Zhang Phys. Rev. E 77, 016406 (2008).

[12] Y. Sentoku, K. Mima, P. Kaw, and K. Nishikawa, Phys. Rev. Lett. 90, 155001 (2003).

[13] A. J. Kemp, Y. Sentoku, V. Sotnikov, and S. C. Wilks, Phys. Rev. Lett. 97, 235001 (2006).

[14] R. J. Mason, Phys. Rev. Lett. 96, 035001 (2006).

[15] J. Honrubia and J. Meyer-ter-Vehn, Nucl. Fusion 46, L25 (2006).

[16] S. Atzeni, A. Schiavi, J. J. Honrubia, X. Ribeyre, G. Schurtz, Ph. Nicolai, M. Olazabal-Loume, C. Bellei, R. G. Evans, and J. R. Davies, Phys. Plasmas 15, 056311 (2008).

[17] C. K. Birdsall and A. B. Langdon, Plasma Physics via Computer Simulation (McGraw-Hill, New York, 1985).

[18] R. W. Hockney and J. W. Eastwood, Computer Simulation Using Particles (McGraw-Hill, New York, 1981).
[19] K. S. Yee, IEEE Trans. Antennas Propag. 14, 302 (1966).

[20] A. R. Bell, J. R. Davies, S. Guerin and H. Ruhl, Plasma Phys. Control. Fusion 39, 653 (1997).

[21] J. R. Davies, Phys. Rev. E 65, 026407 (2002).

[22] L. Gremillet, G. Bonnaud and F. Amiranoff, Phys. Plasmas 9, 941 (2002).

[23] J. J. Honrubia, J. Meyer-ter-Vehn, Plasma Phys. Control Fusion 51, 014008 (2009).

[24] Y. Sentoku and A. J. Kemp, J. Comp. Phys. 227, 6846 (2008).

[25] B. Chrisman,Y. Sentoku, and A. J. Kemp, Phys. Plasmas 15, 056309 (2008).

[26] B. I. Cohen, A. J. Kemp and L. Divol, J. Comp. Phys. 229, 4591 (2010).

[27] S. I. Braginskii, Reviews of Plasma Physics, (M.A. Leontovich vol. 1, Consultants Bureau, New York, page 205, 1965).

[28] W.-M. Wang, P. Gibbon, Z.-M. Sheng, and Y.-T. Li, Phys. Rev. Lett. 114, 015001 (2015).

[29] M. Chen, Z.M. Sheng, J. Zheng, Y.Y. Ma, J. Zhang, Chin. J. Comp. Phys. 25, 43 (2008) (in Chinese).

[30] Y. Sentoku, K. Mima, Y. Kishimoto, M. Honda, J. Phys. Soc. Jpn. 67, 4084 (1998).

[31] T. Takizuka and H. Abe, J. Comput. Phys. 25, 205 (1977).

[32] T. Umeda, Y. Omura, T. Tominaga, and H. Matsumoto, Comput. Phys. Comm. 156, 73 (2003).

[33] T. Z. Esirkepov, Comput. Phys. Comm.135, 144 (2001).

[34] J. W. Eastwood, Comput. Phys. Comm. 64, 252 (1991).

[35] J. Villasenor and O. Buneman, Comput. Phys. Comm. 69, 306 (1992).

[36] M. V. Ammosov, N. B. Delone, and V. P. Krainov, Sov. Phys. JETP 64, 1191 (1986).

[37] B. M. Penetrante and J. N. Bardsley, Phys. Rev. A 43, 3100 (1991).

[38] G. Gibson, T. S. Luk, and C. K. Rhodes, Phys. Rev. A 41, 5049 (1990).

[39] L. V Keldysh, Soy. Phys. JETP 20, 1307 (1965).

[40] W.-M. Wang, Z.-M. Sheng, H.-C. Wu, M. Chen, C. Li, J. Zhang, and K. Mima, Opt. Express 16, 16999 (2008).

[41] W.-M. Wang, S. Kawata, Z.-M. Sheng, Y.-T. Li, J. Zhang, L. M. Chen, L. J. Qian, J. Zhang, Opt. Lett. 36, 2608 (2011).

[42] W.-M. Wang, Y.-T. Li, Z.-M. Sheng, X. Lu, and J. Zhang, Phys. Rev. E 87, 033108 (2013).

[43] W.-M. Wang, P. Gibbon, Z.-M. Sheng, and Y.-T. Li, Phys. Rev. A 90, 023808 (2014).

[44] L. D. Landau and E. M. Lifshitz, "The Classical Theory of Fields", chapter 76. Elsevier, Oxford, 2nd edition (1975). 